\title{
Identification and enumeration of Clostridium spp. In sufu
}

\author{
Xu Qiong ${ }^{1}$, Liu Yang ${ }^{1 *}, Y_{u} \mathrm{Yi}^{1}$, Zhang Nana ${ }^{1}$, Wang Yue ${ }^{1}$, Zhao Lei ${ }^{1}$, Weng Shiyu ${ }^{1}$ \\ ${ }^{1}$ Shanghai Institute of Quality Inspection and Technical Research, Shanghai, China
}

\begin{abstract}
In this study, Clostridium spp. was counted in 49 Chinese commercial sufu samples, and 25 samples were detected Clostridium in all samples: the detection rate was $51.02 \%$. About $93.87 \%$ of the samples contained Clostridium at low levels $(\log \mathrm{CFU} / \mathrm{g}<3.0)$ and one sample had over $4.12 \mathrm{lg} \mathrm{CFU} / \mathrm{g}$ indicating potential hazard to consumers. The $16 \mathrm{~S}$ rDNA identification was carried out on the genus Clostridium, and the isolates were confirmed as Clostridium botulinum, Clostridium sporogenes, Clostridium beijerinckii and Clostridium bifermentans, while Clostridium butyricum, Clostridium botulinum, Clostridium perfringens and Clostridium bifermentans were the species detected in the sufu samples by high-throughput sequencing. Based on these results, the research on the number and distribution of clostridium and the possible risks of biotoxins in fermented food needs to be strengthened. To our knowledge, this is the first study to isolate and identify Clostridium spp. in sufu.
\end{abstract}

\section{Introduction}

Sufu is a kind of traditional fermented bean curd with more than 1000 years of history in China. It is a cheeselike, creamy, soft product made from tofu and fermented by microorganisms. It is also known as "Chinese

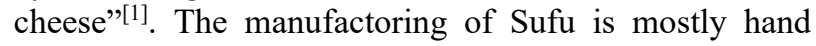
work in a semi-open environment without heat sterilization. Sufu may be contaminated with microbes from the air. This would influence Sufu quality and even increase hazards and risks in food safety ${ }^{[2]}$.

In the previous work, we fund that the relative abundance of clostridium in one sufu sample was $7.12 \%$ by high-throughput sequencing ${ }^{[3]}$.Clostridia are grampositive, spore-forming, anaerobic bacteria which are considered to be the principal organisms responsible for late blowing ${ }^{[4]}$. A number of cases of botulism have been reported for sufu products. In 2017, a botulism outbreak was associated with by eating homemade stinky tofu and self-cured soybean products ${ }^{[5]}$ and in 1998, also a homemade sufu caused an outbreak ${ }^{[6]}$. Inoculation studies with the causative strain showed the growth of Clostridium botulinum and toxin formation in the sufu.

The aim of the study was to count, isolated and identify Clostridium spp. in sufu samples. Illuminebased sequencing with clostridium was to study the diversity of the species. The identification was performed by $16 \mathrm{~S}$ rDNA sequencing, and high throughput sequencing was used to analyze the bacterial flora in sufu samples.

\section{MATERIALS AND METHODS}

\subsection{Sufu samples}

A total of 47 sufu samples were collected from China. Sufu samples were collected from markets in different regions of China (Table1)

Table1 sufu samples collected from variances of china

\begin{tabular}{|c|c|c|c|}
\hline $\begin{array}{l}\text { sample } \\
\text { code }\end{array}$ & $\begin{array}{l}\text { production } \\
\text { location }\end{array}$ & $\begin{array}{l}\text { sample } \\
\text { code }\end{array}$ & $\begin{array}{c}\text { production } \\
\text { location }\end{array}$ \\
\hline $\mathrm{C} 1$ & Beijing & B1 & Hunan \\
\hline $\mathrm{C} 2$ & Beijing & $\mathrm{B} 2$ & Beijing \\
\hline $\mathrm{C} 3$ & Zhejiang & B3 & Zhejiang \\
\hline $\mathrm{C} 4$ & Anhui & B4 & Jiagnsu \\
\hline $\mathrm{C} 5$ & Zhejiang & B5 & Anhui \\
\hline C6 & Zhejiang & B6 & Anhui \\
\hline $\mathrm{C} 7$ & Anhui & B7 & Sichuan \\
\hline $\mathrm{C} 8$ & Shandong & B8 & Sichuan \\
\hline C9 & Heilongjiang & B9 & Guangdong \\
\hline $\mathrm{C} 10$ & Sichuan & B10 & Guangdong \\
\hline $\mathrm{C} 11$ & Jilin & B11 & Guangdong \\
\hline $\mathrm{C} 12$ & Anhui & B12 & Jiagnxi \\
\hline $\mathrm{C} 13$ & Shandong & B13 & Guagnxi \\
\hline H1 & Hunan & B14 & Sichuan \\
\hline $\mathrm{H} 2$ & Beijing & B15 & Guangxi \\
\hline H3 & Beijing & B16 & Beijing \\
\hline H4 & Zhejiang & B17 & Fujian \\
\hline H5 & Jiagnsu & B18 & Sichuan \\
\hline H6 & Guangdong & B19 & Sichuan \\
\hline $\mathrm{H} 7$ & Tianjin & B20 & Guangxi \\
\hline $\mathrm{H} 8$ & Beijing & B21 & Guangxi \\
\hline \multirow[t]{2}{*}{ H9 } & Shanghai & B22 & Zhejiang \\
\hline & & $\mathrm{B} 23$ & Jiangxi \\
\hline
\end{tabular}




$\begin{array}{cc}\text { B24 } & \text { Jiangxi } \\ \text { B25 } & \text { Zhejiang } \\ \text { B26 } & \text { Jiagnsu } \\ \text { B27 } & \text { Shanghai }\end{array}$

\subsection{Microbiological sampling}

The content of Clostridium was analysed according to GB 4789.13-2012. In brief, $25 \mathrm{~g}$ of sample were transferred to nine times the volume (approximately 225 $\mathrm{ml}$ ) of BPW and homogenised in a stomacher for one minute. A 10 -fold serial dilution in 0.1 per cent $(\mathrm{v} / \mathrm{v})$ peptone water (Luqiao) was prepared and $1.0 \mathrm{ml}$ from each dilution was poured onto a Differentia Reinforced Clostridial Agar (DRCA) agar (Haibo) on a petri dish measuring $9 \mathrm{~cm}$ in diameter. An additional $10 \mathrm{ml}$ DRCA was poured as an overlay. All agar plates were incubated in an anaerobic atmosphere generated by the use of MARK II (ANOXOMAT) at $37^{\circ} \mathrm{C}$ for $48 \pm 3$ hours. The number of Clostridium was expressed as log CFU/g.

\subsection{DNA extraction and PCR amplification}

\subsubsection{DNA extraction}

DNA was isolated from $1 \mathrm{ml}$ liquid cultures and from several colonies obtained from agar plates. The DNA was extracted with MiniBEST Bacteria Genomic DNA Extraction Kit Ver.3.0(TaKaRa) according to the manufacturer's instructions. The extracted DNA was frozen at $-20^{\circ} \mathrm{C}$ or directly subjected to PCR analysis.

Sufu genomic DNA was extracted from each sample using Modified CTAB method was used: three samples of the same batch were taken and $50 \mathrm{~mL}$ Sufu soup was poured from each sample, totaling $150 \mathrm{~mL}$. After mixing, the mixed sample soup was centrifuged for $10 \mathrm{~min}$ at $9000 \mathrm{rpm}$, then the supernatant was removed, and the sediment was added with $10 \mathrm{~mL}$ of sterile water. The supernatant was removed by centrifugation at $9000 \mathrm{rpm}$ for $10 \mathrm{~min}$. The supernatant was added with $800 \mu \mathrm{L}$ lysozyme $(10 \mathrm{mg} / \mathrm{mL})$ and the sediment was bathed in water at $37 \mathrm{C}$ for $1 \mathrm{~h}$. Then $40 \mathrm{mu} \mathrm{L}$ protease $\mathrm{K}$ and CTAB lysate were added to the sediment, and the water bath was held at $56 \mathrm{C}$ for 2 hours. Tris saturated phenol/trichloromethane/isoamyl alcohol with the ratio of $25: 24: 1$ was added to the cracking solution of each tube. The mixture was centrifuged at $13000 \mathrm{rpm}$ for 10 min. The supernatant was put into the new centrifugal tube, added with trichloromethane: isoamyl alcohol (24:1) of same volume, mixed well, centrifuged at 13000 $\mathrm{rpm}$, for $10 \mathrm{~min}$. The supernatant was moved to the new centrifugal tube and mixed with 2-fold volume of anhydrous ice ethanol. The supernatant was kept for about 30 minutes, then centrifuged at $13000 \mathrm{rpm}$, for 2 minutes. The supernatant was discarded and precipitated 2-3 times with $70 \%$ ethanol. The precipitation was placed at room temperature for natural air drying. After air drying, $50 \mathrm{~L}$ TE solution was added to dissolve the precipitation.

\subsubsection{Illumina MiSeq sequencing}

Purified DNA was used as the template for PCR amplification for the bacterial 16S rRNA genes. The V1V3 variable regions of the bacterial 16S rRNA gene were amplified by the primer pair $27 \mathrm{~F}$ (5'AGAGTTTGAT CCTGGCTCAG-3') and 515R (5'ATTACCGCGGCTGCTGG -3') , and the internal transcribed spacer 2 (ITS 2) region of fungi amplicons was amplified with forward primer ITS2F (5'GCATCGATGAAGAACGCAGC-3') and reverse primer ITS2R (5'- TCCTCCGCTTATTGATATGC-3') primers. The PCR conditions were as follows: initial denaturation at $94^{\circ} \mathrm{C}$ for 2 minutes; denaturation at $94^{\circ} \mathrm{C}$ for 30 seconds, annealing at $60^{\circ} \mathrm{C}$ for 50 seconds; extension at $72^{\circ} \mathrm{C}$ for 45 seconds, amplification for 40 cycles; preservation at $4^{\circ} \mathrm{C}$. The quality of the amplified PCR products was checked by electrophoresis in $1 \%$ agarose gel. Each primer was binded different barcodes and the same adaptors. PCR amplicons were purified and sequenced on the IlluminaMiSeq platform.

\subsection{Processing of high-throughput sequencing data[7]}

\subsubsection{Pre-process}

FASTQC(http://www.bioinformatics.babraham.ac.uk/pr ojects/fastqc) is used for the quality control to check on the quality of raw data. Because the data is generated by Illumina, the universal adaptor from the raw data should be removed by CUTADAPT (http://code.google.com/p/cutadapt/). Pair end reads are joined together using FLASH (https://sourceforge.net/projects/flashpage/files/). Reads are de-multiplexed using the Quantitative Insights Into Microbial Ecology (QIIME)pipeline. First, using "split_libraries_fastq.py" which performs demultiplexing of Fastq sequence data where barcodes and sequences are contained in two separate fastq files above (common on Illumina runs) to get a fasta file which contains the sequence of 16S rRNA and a fasta file of the quality of the sequence file. Removing the chimera sequences in the data. Using "identify_chimeric_seqs.py" which identify chimeric sequences in input $\overline{\text { FASTA file with }}$ usearch61 algorithm to identify the chimera and using "filter_fasta.py" module to remove the chimeric sequences.

\subsubsection{Clean data Analysis}

Sequences were grouped into operational taxonomic units (OTUs) at $97 \%$ sequence similarity using the Greengenes reference database. OTUs that did not cluster with known taxa at $97 \%$ identity or higher in the database were clustered de novo and not de novo $(\text { UCLUST) })^{[8]}$. 


\subsection{Statistical analysis}

All experiments were based on a completely randomized block designs, and were performed in triplicate. Results were presented as mean \pm standard deviation(SD) of replicated measurements.

Table 2. Microbiological composition of commercial sufu (log CFU/g sample)

\begin{tabular}{|c|c|c|c|c|c|}
\hline Sample & Clostridium & Sample & Clostridium & Sample & Clostridium \\
\hline H1 & $<1$ & $\mathrm{C} 1$ & $<1$ & B1 & $1.66 \pm 0.21$ \\
\hline $\mathrm{H} 2$ & $2.02 \pm 0.30$ & $\mathrm{C} 2$ & $<1$ & B2 & $<1$ \\
\hline H3 & $2.06 \pm 0.46$ & $\mathrm{C} 3$ & $2.98 \pm 0.16$ & B3 & $<1$ \\
\hline $\mathrm{H} 4$ & $2.45 \pm 0.34$ & $\mathrm{C} 4$ & $<1$ & B4 & $<1$ \\
\hline H5 & $<1$ & $\mathrm{C} 5$ & $1.26 \pm 0.24$ & B5 & $<1$ \\
\hline H6 & $<1$ & C6 & $1.10 \pm 0.17$ & B6 & $<1$ \\
\hline $\mathrm{H} 7$ & $1.64 \pm 0.19$ & $\mathrm{C} 7$ & $<1$ & B7 & $<1$ \\
\hline H8 & $1.10 \pm 0.17$ & $\mathrm{C} 8$ & $1.83 \pm 0.56$ & B8 & $<1$ \\
\hline \multirow[t]{19}{*}{ H9 } & $1.39 \pm 0.12$ & C9 & $1.00 \pm 0.00$ & B9 & $3.02 \pm 0.59$ \\
\hline & & $\mathrm{C} 10$ & $3.15 \pm 0.11$ & B10 & $<1$ \\
\hline & & $\mathrm{C} 11$ & $<1$ & B11 & $1.98 \pm 0.55$ \\
\hline & & $\mathrm{C} 12$ & $<1$ & B12 & $1.10 \pm 0.17$ \\
\hline & & $\mathrm{C} 13$ & $4.12 \pm 0.03$ & B13 & $1.08 \pm 0.19$ \\
\hline & & & & B14 & $<1$ \\
\hline & & & & B15 & $1.23 \pm 0.40$ \\
\hline & & & & B16 & $<1$ \\
\hline & & & & B17 & $<1$ \\
\hline & & & & B18 & $2.04 \pm 0.15$ \\
\hline & & & & B19 & $<1$ \\
\hline & & & & B20 & $1.00 \pm 0.00$ \\
\hline & & & & B21 & $1.00 \pm 0.00$ \\
\hline & & & & B22 & $2.80 \pm 0.37$ \\
\hline & & & & B23 & $<1$ \\
\hline & & & & B24 & $<1$ \\
\hline & & & & B25 & $<1$ \\
\hline & & & & B26 & $2.04 \pm 0$ \\
\hline & & & & B27 & $1.00 \pm 0$ \\
\hline
\end{tabular}

From Table 2, 51.02\% of the samples contained Clostridium spp. were detected in 49 Sufu samples. Among them, the detection rate of red sufu, white sufu and gray sufu was $66.67 \%, 44.44 \%$ and $58.85 \%$ respectively. (24 products, no isolates could be made with the present methodology. The number of Clostridium spp. varied between 1.00 and $4.12 \log$ CFU/g. About $93.87 \%$ of the samples contained Clostridium at low levels $(\log \mathrm{CFU} / \mathrm{g}<3.0)$, but sample B9 $\mathrm{C} 10$ and $\mathrm{C} 13$ contained $3.02 \pm 0.59,3.15 \pm 0.11$ and $4.12 \pm 0.03 \log \mathrm{CFU} / \mathrm{g}$ respectively.

Bacterial colonies were isolated and purified from the growing and counting plate specimens and identified by 16S rDNA. By Blast comparison, the suspected bacteria were mainly identified as: Clostridium beijerinckii, Clostridium bifermentans, Clostridium botulinum and Clostridium sporogenes.

\section{RESULTS AND DISCUSSION}

\subsection{Microbiological analysis}


Clostridium botulinum and Clostridium bifermentans were detected by High throughput sequencing: the results are consistent to $16 \mathrm{~s}$ rDNA sequencing. Clostridium perfringens is a common pathogen, high relative abundance was found in B15, B22 and $\mathrm{C} 5$ samples, respective $67.52 \%, 29.73 \%$ and $56.52 \%$.

Table 3. Relative abundance of Clostridium in sufu samples

\begin{tabular}{ccccccc}
\hline & Clostridium spp & Cl.butyricum & Clostridium; Other & Cl.botulinum & Cl.perfringens & Cl.bifermentans \\
\hline B7 & $0.01 \%$ & $100 \%$ & 0 & 0 & 0 & 0 \\
B10 & $0.01 \%$ & $100 \%$ & 0 & 0 & 0 & 0 \\
H1 & $0.06 \%$ & $100 \%$ & 0 & 0 & 0 & 0 \\
B15 & $7.00 \%$ & $0.34 \%$ & $5.04 \%$ & $26.58 \%$ & $67.52 \%$ & $0.51 \%$ \\
B22 & $0.18 \%$ & $16.22 \%$ & $43.24 \%$ & $10.81 \%$ & $29.73 \%$ & 0 \\
H2 & $0.02 \%$ & $100 \%$ & 0 & 0 & 0 & 0 \\
H3 & $0.01 \%$ & $100 \%$ & 0 & 0 & 0 & 0 \\
H5 & $0.02 \%$ & $0 \%$ & $100 \%$ & 0 & 0 & 0 \\
H7 & $0.01 \%$ & $100 \%$ & 0 & 0 & 0 & 0 \\
H8 & $2.13 \%$ & $95.07 \%$ & $1.57 \%$ & $2.24 \%$ & $1.12 \%$ & 0 \\
C5 & $0.16 \%$ & $8.70 \%$ & $4.35 \%$ & $30.43 \%$ & $56.52 \%$ & 0 \\
C6 & $0.01 \%$ & 0 & $50.00 \%$ & 0 & 0 & $50.00 \%$ \\
C7 & $2.14 \%$ & $2.93 \%$ & $17.95 \%$ & $6.59 \%$ & $2.56 \%$ & $69.96 \%$ \\
C8 & $0.10 \%$ & $30.00 \%$ & $30.00 \%$ & $20.00 \%$ & $20.00 \%$ & 0 \\
\hline
\end{tabular}

However, We did not identify Clostridium perfringens in the all samples by $16 \mathrm{~S}$ rDNA, similar to Clostridium butyric. The results showed that there were some differences between the results of high-throughput sequencing and traditional microbial culture identification methods. Because of the unsuitable culture conditions, some microorganisms cannot be screened by traditional methods.

Due to the special production process of sufu, it is possible to be contaminated by Clostridium. In this study, the Clostridium species in the samples were counted, and the virulence types of the isolates were not studied in depth. In addition, because most of the Clostridium is fastidious bacteria, the culture cycle is long and the spores are easy to form, so the common bacterial identification methods are not easy to obtain satisfied results. There may still be other species of Clostridium that have not been cultured. However, it is necessary to pay attention to the existence of these bacteria in sufu. The number, distribution and biotoxin of Clostridium need to be strengthened.

\section{REFERENCES}

1. K H Steinkraus. Handbook of indigenous fermented foods. 2nd ed., rev. and expanded.[J]. Microbiology, 1996,62,3,131.

2. Yu Shizhe, Fan Yi, Han Beizhong, et al. Analysis of microorganism during the production and processing environment of low-salt sufu[J]. China Brewing, 2017,36,12,18-22.

3. XU Qiong, LIU Yang, QU Qinfeng, et al. Highthroughput sequencing analysis of bacterial diversity in red sufu from different regions[J]. Food Science, 2020,10,41,110-116.
4. Cremonesi P, Vanoni L, Silvetti T, et al. Identification of Clostridium beijerinckii, $\mathrm{Cl}$. butyricum, $\mathrm{Cl}$. sporogenes, $\mathrm{Cl}$. tyrobutyricum isolated from silage, raw milk and hard cheese by a multiplex PCR assay[J]. Journal of Dairy Research, 2012,79,3,318-323.

5. Chang Wenguang, Liu Yi, Liu Peng, et al. Clinical analysis of 5 cases of botulinum toxin B poisoning [J]. Journal of Clinical Neurology, 2017,1,

6. Li Bangchuan, Chen Nanjun, Li Yong. An investigation report of botulism caused by homemade fermented bean curd [J]. PLA Journal of Preventive Medicine, 1998.

7. Caporaso JG, Kuczynski J, Stombaugh J, et al. QIIME allows analysis of high-throughput community sequencing data[J]. Nat Methods, 2010,4,5,335-336.

8. C E R. Search and clustering orders of magnitude faster than BLAST[J]. Bioinformatics, 2010,26,19,2460-2461. 